\title{
Severe mitral regurgitation recurrence after successful percutaneous mitral edge-to-edge repair by Mitraclip: Insights from a three-dimensional echocardiography study
}

Yoan Lavie Badie ${ }^{1}$, Fabien Vannier ${ }^{1}$, Eve Cariou $^{1}$, Pauline Fournier ${ }^{1}$, Romain Itier ${ }^{1}$, Stéphanie Cazalbou ${ }^{1}$, Virginie Blanchard ${ }^{1}$, Michel Galinier ${ }^{1}$, Didier Carrie ${ }^{1}$, Olivier Lairez $^{1}$, Frédéric Bouisset ${ }^{1}$, and Thibault Lhermusier $^{1}$

${ }^{1}$ Hopital de Rangueil

January 27, 2021

\begin{abstract}
Background: The sustainability of the results of mitraclip procedures is a source of concern. Aims: To investigate risk factors of severe mitral regurgitation (MR) recurrence after Mitraclip in primary MR. Methods and results: Eighty-three patients undergoing successful Mitraclip procedures were retrospectively included. Valve anatomy and Mitraclips placement were comprehensively analyzed by post-processing $3 \mathrm{D}$ echocardiographic acquisition. The primary composite endpoint was the recurrence of severe MR. Mean age was $83 \pm 7$ years-old, 37 (44\%) were female. Median follow-up was 381 days (IQR 195-717) and $17(20 \%)$ patients reached the primary endpoint. Main causes of recurrence of severe MR were relapse of a prolapse (64\%) and single leaflet detachment (23\%). Posterior coaptation line length (HR 1.06 95\%CI 1.01-1.12 p=0.02), poor imaging quality (HR 3.84, 95\%CI1.12-13.19; $\mathrm{p}=0.03$ ), and inter-clip distance (HR 1.60, 95\%CI 1.27-2.02; $\mathrm{p}<0.01$ ) were associated with the occurrence of the primary endpoint. Conclusions: Recurrence of severe MR after a MitraClip procedure for primary MR is common and results from a complex interplay between anatomical (tissue excess) and procedural criteria (quality of ultrasound guidance and MitraClips spacing).
\end{abstract}

\section{Hosted file}

MRrecurrent2.pdf available at https://authorea.com/users/392051/articles/506063-severemitral-regurgitation-recurrence-after-successful-percutaneous-mitral-edge-to-edgerepair-by-mitraclip-insights-from-a-three-dimensional-echocardiography-study
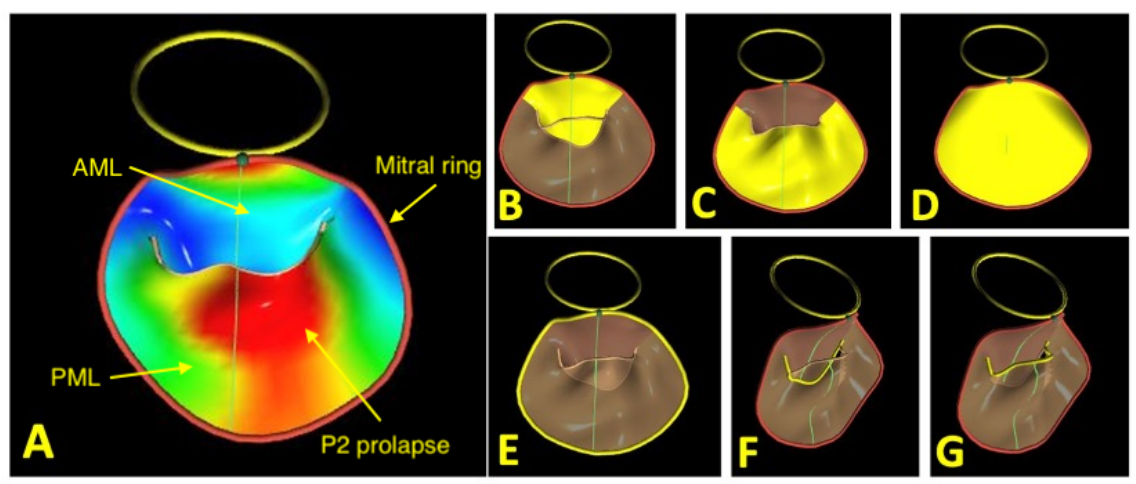

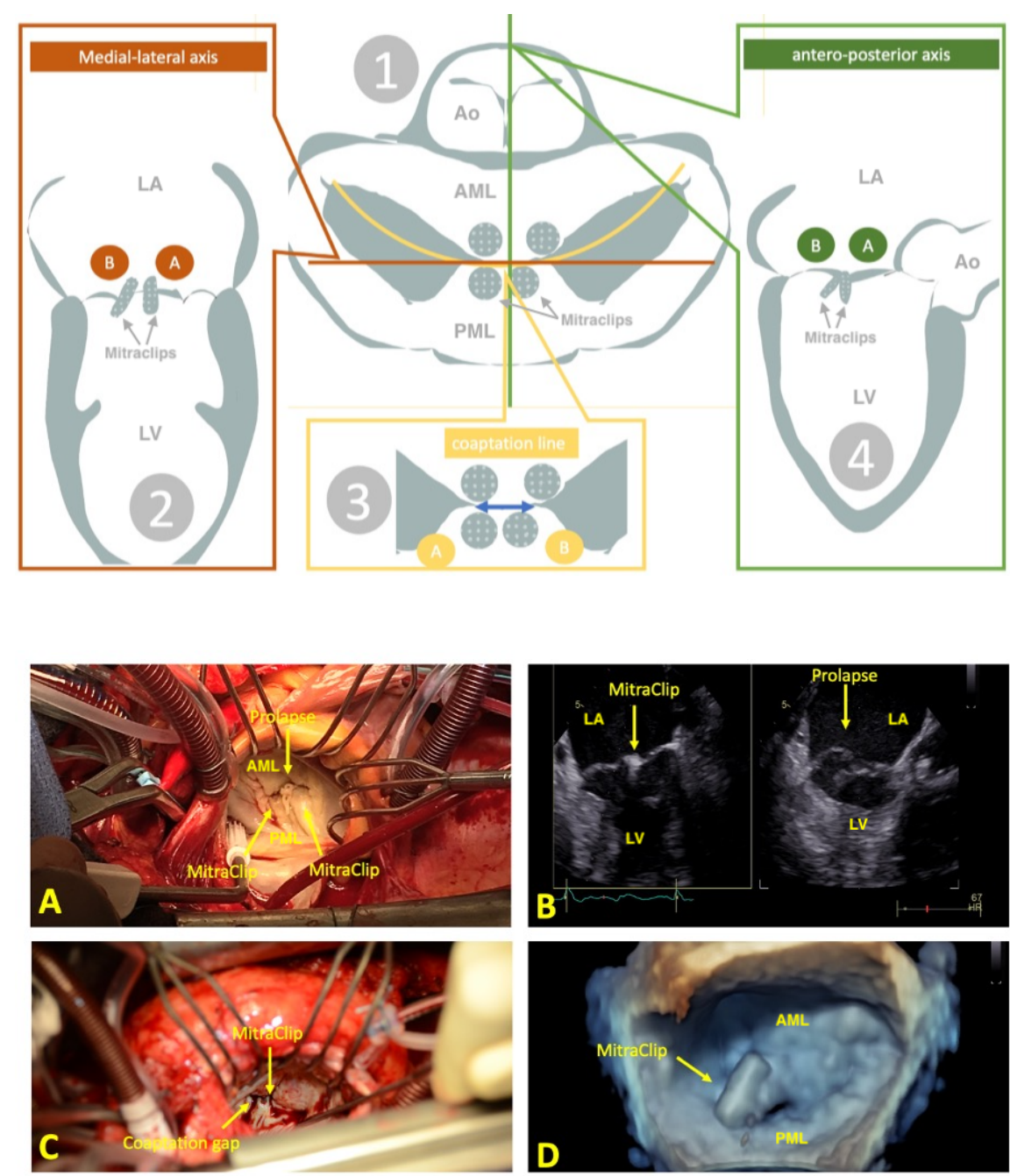